\title{
ÍNDICE E FATORES DE RISCOS PARA DIABETES MELLITUS EM UM GRUPO DE IDOSOS DE MINEIROS-GO
}

\author{
Diabetes Mellitus Index And Risk Factors In An Elderly Group Of Mineiros-Go
}

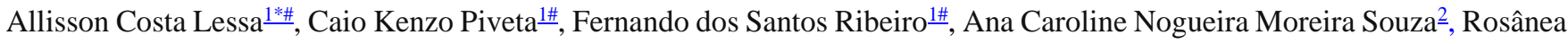
Meneses de Souza ${ }^{\underline{3}}$

\begin{abstract}
RESUMO
Esta pesquisa teve como objetivo verificar o índice e os principais fatores de riscos, para o diabetes mellitus, que podem acometer em um grupo de idosos, em Mineiros-GO. Trata-se de um estudo quantitativo, do tipo levantamento de dados. Os participantes da pesquisa foram idosos com idade mínima de 60 anos que aceitaram participar do estudo, no período de agosto e setembro de 2019. Foram entrevistados 56 idosos e os dados obtidos foram analisados no programa Microsoft Office Excel 2016. A maioria dos participantes foram mulheres com idade entre 66 a 70 anos, de etnia parda/mulata. Quanto a atividade física, $62 \%$ dos idosos praticam, pelo menos, duas vezes por semana. Em relação ao hábito alimentar, a maioria possui uma dieta regularmente saudável para alimentos naturais. Porém, ao analisar a alimentação baseada em alimentos gordurosos, embutidos e frituras, 59\% dos idosos relataram consumir, pelo menos, duas vezes na semana. O consumo de refrigerantes e bebidas alcoólicas, foi considerado moderado/baixo. Um dado preocupante foi a frequência do consumo de alimentos ricos em carboidratos, $56 \%$ dos idosos consomem de duas a três vezes na semana. Consequentemente, $82 \%$ dos entrevistados estão acima do peso, de acordo com o cálculo do índice de massa corporal. Com os resultados deste trabalho, é possível concluir que o fator de risco genético se mostrou relevante e o Índice de Massa Corporal da maioria está acima do peso ou em obesidade I. É válido ressaltar, que a prevalência de diabetes mellitus, mesmo sendo menor em homens, não é condizente com o curso clínico desta, pois são neles a prevalência dos casos mais graves e com pior prognóstico da doença, visto que não possuem hábitos para o controle e manejo desta morbidade clínica.

Palavras-chave: Diabetes mellitus II; População idosa; Fatores de risco.
\end{abstract}

\section{ABSTRACT}

This research aimed to verify the index and the main risk factors for diabetes mellitus, which can affect a group of elderly people in Mineiros-GO. This is a quantitative study, of the data collection type. Research participants were elderly aged at least 60 years who agreed to participate in the study, in the period from August to September 2019. 56 elderly were interviewed and the data obtained were analyzed using the Microsoft Office Excel 2016 program. Most participants were women aged between 66 and 70 years, of mixed race/mulatto. As for physical activity, $62 \%$ of the elderly practice at least twice a week. Regarding eating habits, most have a regularly healthy diet of natural foods. However, when analyzing the diet based on fatty foods, sausages and fried foods, $59 \%$ of the elderly reported consuming at least twice a week. The consumption of soft drinks and alcoholic beverages was considered moderate/low. A worrying fact was the frequency of consumption of foods rich in carbohydrates, $56 \%$ of the elderly consume two to three times a week. Consequently, $82 \%$ of respondents are overweight, according to the calculation of the body mass index. With the results of this work, it is possible to conclude that the genetic risk factor proved to be relevant and the Body Mass Index of the majority is overweight or obese I. It is worth noting that the prevalence of diabetes mellitus, even being lower in men, is not consistent with the clinical course of this, as they are the prevalence of the most severe cases and with the worst prognosis of the disease, as they do not have habits to control and manage this clinical morbidity.

Keywords: Diabetes mellitus II; Elderly population; Risk factors.

1. Acadêmico do curso de Medicina da Faculdade Morgana Potrich (FAMP), Mineiros-GO, Brasil.

2. Especialista na área de Medicina em Saúde da Família, Preceptoria e Clínica Médica, Preceptora do curso de Medicina pela Faculdade Morgana Potrich - FAMP. Goiânia-GO, brasil

3. Bióloga, Mestre em Biologia pela Universidade Federal de Mato Grosso do Sul, Campo Grande, MS. Coordenadora de Pesquisa na FAMP - Faculdade Morgana Potrich, Mineiros-GO, Brasil.

*Autor para Correspondência. E-mail: allissonlessa@ gmail.com

\#Mesma contribuição autoral. 


\section{INTRODUÇÃO}

O diabetes mellitus (DM) é caracterizada como um distúrbio metabólico que favorece a deficiência ou a ausência de insulina, causando quadros de alterações glicêmicas prejudiciais ao organismo. Assim, é classificada em dois principais tipos, a DM Tipo 1, que é conhecida como insulinodependente, onde acontece a deficiência absoluta de insulina, e também a DM Tipo 2, que ocorre uma diminuição da secreção de insulina ou casos onde o corpo desenvolve resistência a mesma. Com isso, alterações relevantes sobre este hormônio possibilitam os quadros hiperglicêmicos desenvolvidos por esta patologia. ${ }^{1,2,3}$

De acordo com o $9^{\circ}$ Atlas de Diabetes, feito pela Federação Internacional de Diabetes, no Brasil, o quadro de portadores de DM chega a valores abundantes de 16,5 milhões de pessoas com a doença, sendo que a metade desconhece o diagnóstico, em 2019. Esses valores, tendem a aumentar com o passar dos anos. ${ }^{4,5,6}$ Dessa forma, segundo a Secretaria de Saúde do Estado de Goiás, o tipo 2 de DM é a mais prevalente, alcançando cerca de $90 \%$ das pessoas diagnosticadas. ${ }^{7}$

Nesse contexto, o acompanhamento do público alvo deste trabalho, é justificado por consequência das necessidades que são ofertadas após o diagnóstico da doença. Desse modo, principalmente os idosos, há, adversidades a serem enfrentadas por conta do avanço da idade, porém, associada às obrigações necessárias para o controle de uma doença crônica.

O diabetes é uma patologia crônica que deve ser avaliada periodicamente pelo profissional da saúde, visando o bem estar populacional e, dessa forma, o estudo busca acompanhar um grupo definido de idosos com a intenção de orientar e melhorar a qualidade de vida destes, ademais pode contribuir para o meio cientifico com os resultados obtidos nas pesquisas, pois, porém é uma doença de alta prevalência e alta morbimortalidade em pessoas de idade avançada o estado de Goiás apresenta poucas referências sobre o caso.

Ao definir um tema, independente da área de conhecimento, deve-se considerar o ponto de vista ético e refletir sobre ideias e conceitos mutáveis tanto de uma sociedade para outra, como no decorrer do tempo. Uma vez que este trabalho envolve atitudes influenciáveis por usos e costumes, veiculadas tanto pela tradição de geração para geração, como pela mídia, e que sofrem alterações com os avanços científicos e tecnológicos. Por isso, faz-se necessário a descoberta e acompanhamento de determinadas condutas para verificação de suas qualidades como boa ou ruim, como o hábito alimentar e a frequência de atividades físicas.
Neste sentido, esta pesquisa tem como objetivo verificar o índice e os principais fatores de riscos, para o diabetes mellitus, que podem acometer a um grupo de idosos em Mineiros-GO.

\section{MATERIAL E MÉTODOS}

Trata-se de um trabalho quantitativo, do tipo levantamento de dados. Os participantes da pesquisa foram idosos com idade mínima de 60 anos que aceitaram participar do estudo, no período de agosto e setembro de 2019. Foram excluídos participantes com idade abaixo de 60 anos e aqueles que se recusaram assinar o Termo de Consentimento Livre e Esclarecido.

A coleta de dados foi realizada através de entrevistas sigilosas, preservando o anonimato dos participantes. Todos os idosos foram esclarecidos sobre a importância e objetivo do trabalho e, logo após, foram entrevistados, individualmente, utilizando formulário eletrônico.

Foram abordados 68 idosos, desses apenas 56 foram considerados aptos a participar. Os demais se recusaram ou não souberam responder todas as perguntas. Os dados obtidos foram analisados no programa Microsoft Office Excel 2016, utilizando fórmulas para cálculo de médias e desvio padrão.

\section{RESULTADOS E DISCUSSÃO}

$\mathrm{Na}$ figura 1, a qual ilustra a presença de pessoas do sexo feminino e masculino correlacionadas com a sua faixa etária, mostra a prevalência de pessoas do sexo feminino nos programas de extensão, onde o trabalho foi instituído e feito a entrevista.

É notório dizer que, as mulheres preocupam-se mais com as questões direcionadas a sua saúde, e dessa forma, possuem mais adesão ao grupo de extensão, o que justifica sua prevalência acentuada, quando comparadas ao grupo do sexo masculino, que apesar de menor quantidade, possuem a forma de doença mais intensificada e com pior prognostico. ${ }^{8}$

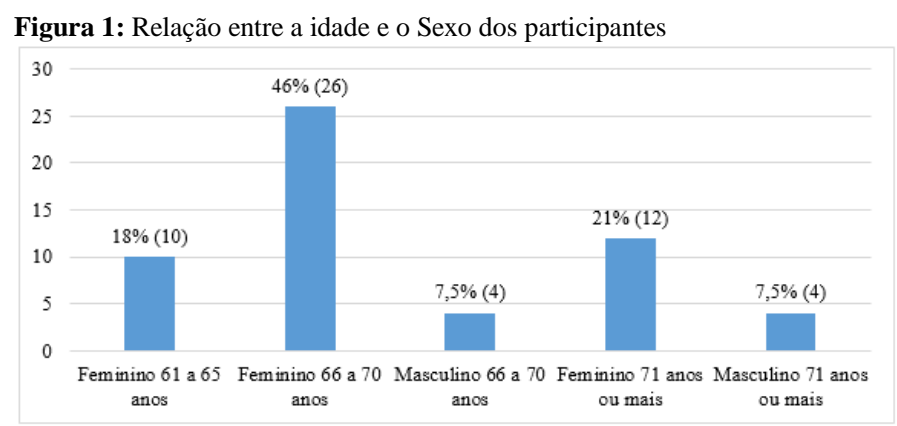

Fonte: os próprios autores. 
A idade do público feminino em torno de 61 a 65 anos chegou em torno de $18 \%$, a idade do público feminino em torno de 66 a 70 anos chegou em torno de 53\%, a idade do público masculino em torno de 66 a 70 anos chegou em torno de 7,5\%, a idade do público feminino em torno de 71 anos chegou em torno de $29 \%$ e a idade do público masculino em torno de 71 anos chegou em torno de 7,5\%.

Com isso, é valido ressaltar a diabetes mellitus se torna cada vez mais presente entre pessoas que possuem idade superior a 55 anos. Fato este, justificável, porque, o aumento da faixa etária faz com que aumente os índices de prevalência das pessoas adquirirem alguma morbidade ou doenças crônicas, ou doenças de caráter crônicodegenerativo. ${ }^{9}$

$\mathrm{Na}$ figura 2, foi correlacionado o sexo e etnia dos entrevistados. Desse modo, é visível que a prevalência de casos do sexo feminino e de cor pardo/mulato foi exorbitantemente maior (33 casos), quando comparado a todos os outros casos, independente de sexo ou raça (23 casos). Com isso, é evidente, que assim como na literatura, os dados da prevalência foram comprobatórios quando comparadas as etnias, pois entre as mulheres, foi evidente que os dados mostraram a relevância da DM entre a raça parda/mulata, quando comparadas em casos de pessoas brancas.

A etnia dos participantes do público feminino com a etnia branca $20 \%$, a etnia dos participantes do público masculino com a etnia branca 7\%, a etnia dos participantes do público feminino com a etnia negra $7 \%$, a etnia dos participantes do público feminino com a etnia parda e ou mulato $59 \%$ e a etnia dos participantes do público masculino com a etnia parda e ou mulato $7 \%$.

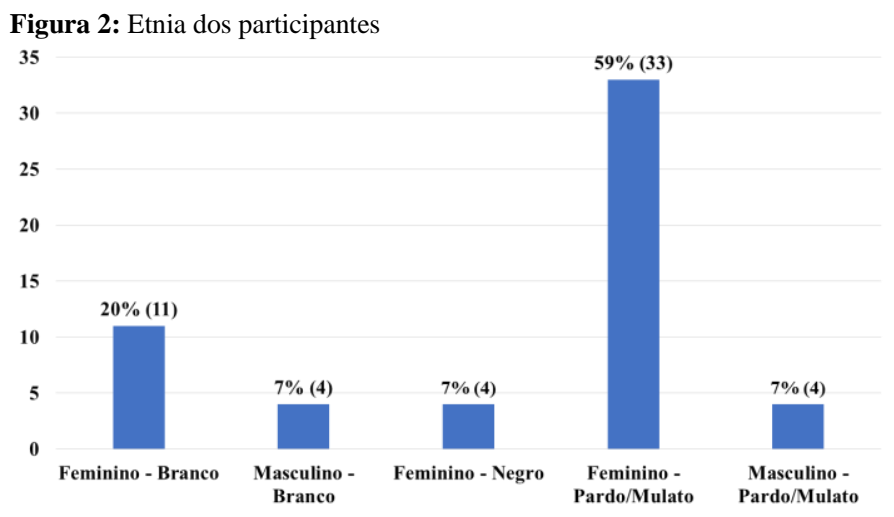

Fonte: os próprios autores.

Isso mostra que, a raça, mesmo que autodeclarada, representa uma característica fenotípica dos indivíduos, e assim, interferem em uma construção de condições socioculturais no contexto que esteja envolvida essas pessoas. Desse modo, estudos feitos com norte-americanos, pessoas que se declaram pardas/negras/mulatas, tendem a serem desempregados, encarcerados ou pobres, o que remetem a possuírem uma condição de vida inferior a dos brancos, justificando a presença de uma vida menos balanceada, quando comparadas as suas alimentações, fator de risco comprovado contra a DM. ${ }^{10}$

$\mathrm{Na}$ figura 3, quando se compara a pratica de atividade física, novamente as mulheres se sobressaem quando comparadas aos homens, pois elas demostram o querer de desenvolver uma vida mais saudável e com menos morbidades do que eles. Isso remete ao fato de que os homens possuem formas de DM mais intensas e com maiores complicações que elas, pois o sedentarismo é um fator de risco alarmante para com a DM, porque contribui para a elevação de colesteróis prejudiciais à saúde, ainda mais quando não se correlaciona a uma alimentação balanceada e adequada para o indivíduo de forma individualizada.

Prática de atividade física realizada pelo público feminino fica em torno de $4 \%$, a prática de atividade física $1 \mathrm{x}$ na semana realizada pelo público feminino fica em torno de $34 \%$, a prática de atividade física $2 \mathrm{x}$ na semana realizada pelo público masculino fica em torno de $34 \%$, a prática de atividade física $3 \mathrm{x}$ na semana realizada pelo público feminino fica em torno de $28 \%$, a prática de atividade física $3 x$ na semana realizada pelo público masculino fica em torno de $4 \%$, a prática de atividade física $4 \mathrm{x}$ na semana realizada pelo público feminino fica em torno de $20 \%$ e a prática de atividade física $4 \mathrm{x}$ na semana realizada pelo público masculino fica em torno de $1 \%$.

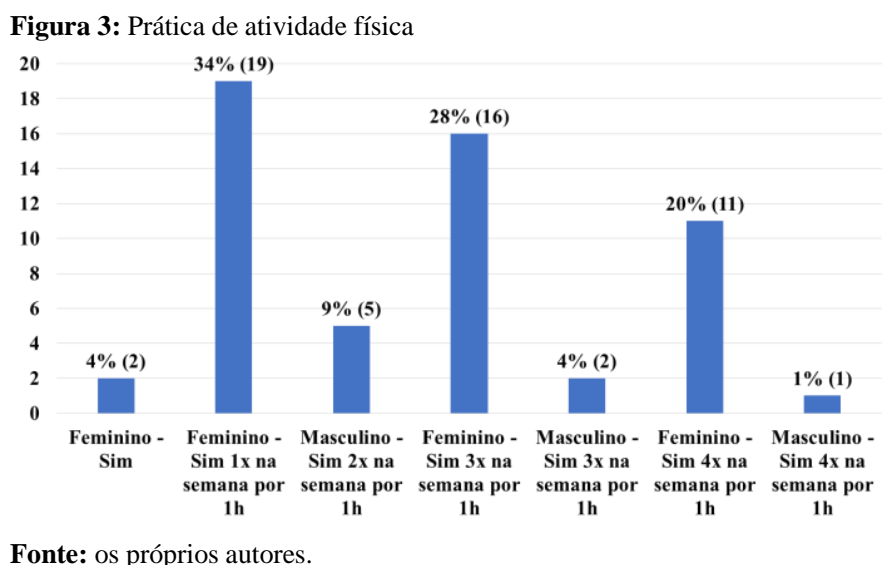

Mesmo quando comparadas as suas práticas, elas possuem grande interesse em desenvolver condições que aumentem a sua sobrevida de uma forma mais saudável, pois 
procuram praticar atividade física regularmente, mesmo que não possam, com compromissos exigidos da vida adulta. 8,3

$\mathrm{Na}$ figura 4, as mulheres, novamente, sobressaem quando comparadas ao público feminino, mesmo que avaliadas em maior quantidade quanto aos homens, pois estas, costumam possuir uma ingesta de alimentos ricos em fibras e frutas, por exemplo, mais acentuada que os homens, que por sua vez, possuem uma alimentação rica em carboidratos distribuídas em todas as suas refeições diárias. Dessa forma, se torna evidente a presença do risco cardiovascular mais prevalente entre os homens, pois o acumulo de gorduras contribui para o aumento de colesteróis não desejados ao organismo quando em combate da DM.

De duas a três vezes fazem o consumo médio, semanal, de alimentos - Frutas de 55\% do público feminino, de duas a três vezes fazem o consumo médio, semanal, de alimentos - Frutas de $9 \%$ do público masculino, de quatro a seis vezes fazem o consumo médio, semanal, de alimentos Frutas de $20 \%$ do público feminino, de quatro a seis vezes fazem o consumo médio, semanal, de alimentos - Frutas de $4 \%$ do público masculino, todos os dias fazem o consumo médio, semanal, de alimentos - Frutas de 10,5\% do público feminino e todos os dias fazem o consumo médio, semanal, de alimentos - Frutas de 1,5\% do público masculino.

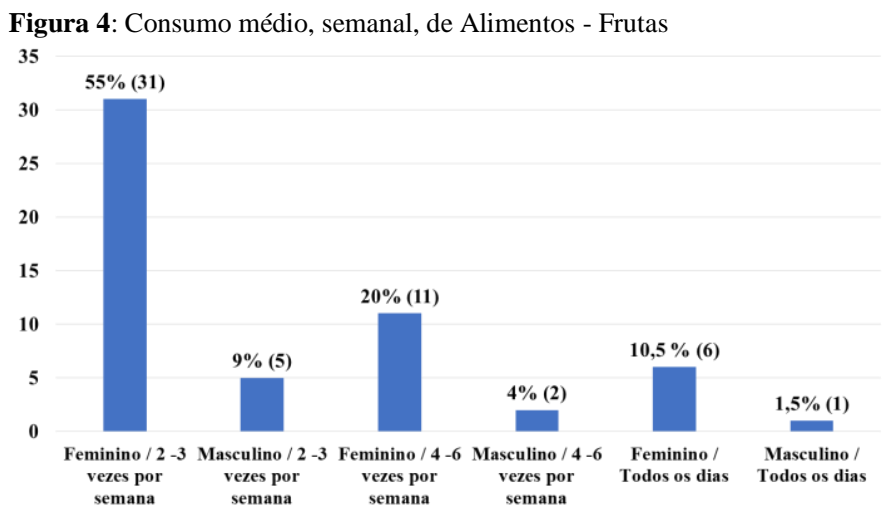

Fonte: os próprios autores.

Nesse contexto, homens, apesar de minoria, representam o maior risco quando comparados quanto a alimentação das mulheres, pois não possuem e não tem desejo de estabelecer uma alimentação saudável. Com isso, estes, são mais susceptíveis a desenvolver as conhecidas placas de ateroma s na parede dos vasos/veias/artérias do corpo, além de suas complicações clinicas, como o AVE (Acidente Vascular Encefálico) ou o IAM (Infarto Agudo do Miocárdio). ${ }^{7}$

$\mathrm{Na}$ figura 5, é evidente a acentuada presença de mulheres que consomem verduras e legumes todos os dias, em detrimento, os homens condensam-se em uma pequena parcela, fazendo o consumo esporadicamente, na maioria dos casos. Dessa forma, vale-se ressaltar a importância do consumo de verdura e legume em ambos os sexos, pois está nesta fonte um dos cuidados com o metabolismo diário de qualquer pessoa, pois este deve ser capaz de suprir de $9-12 \%$ da energia consumida diária.

De duas a três vezes por semana fazem o consumo de verduras e legumes de $13 \%$ do público feminino, de duas a três vezes por semana fazem o consumo de verduras e legumes de $4 \%$ do público masculino, de quatro a seis vezes por semana fazem o consumo de verduras e legumes de $17 \%$ do público feminino, todos os dias fazem o consumo de verduras e legumes de $55 \%$ do público feminino e todos os dias fazem o consumo de verduras e legumes de $7 \%$ do público masculino.

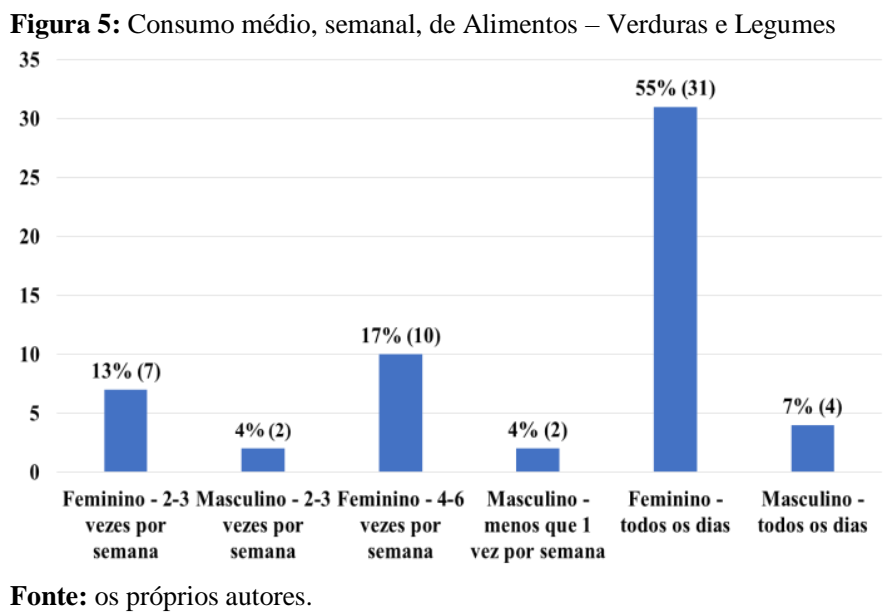

Além disso, o consumo de verduras e legumes, visa um estabelecimento de uma vida saudável e equilibrada, onde riscos cardiovasculares decorrentes do excesso de lipídios/gorduras sejam afastados. Isso remete a importância de manter uma vida baseada em cuidados, principalmente, quando diagnosticadas com DM, uma morbidade crônica que predispõe a pessoa não tratada e sem cuidados a diversos problemas sistêmicos. ${ }^{11}$

Seguindo a entrevista, com as questões relacionadas com a alimentação, a sexta figura aborda a relação do sexo dos participantes com o consumo médio, semanal, de alimentos. É valido ressaltar que $4 \%$ do público feminino realiza o consumo médio de feijão em torno de duas a três vezes por semana, $1,5 \%$ do público feminino realiza o consumo médio de feijão em torno de quatro a seis vezes por semana, $4 \%$ do público masculino realiza o consumo médio de feijão em torno de duas a três vezes por semana, $80 \%$ do público feminino realiza o consumo médio de feijão todos os 
dias e $10,5 \%$ do público masculino realiza o consumo médio de feijão todos os dias.

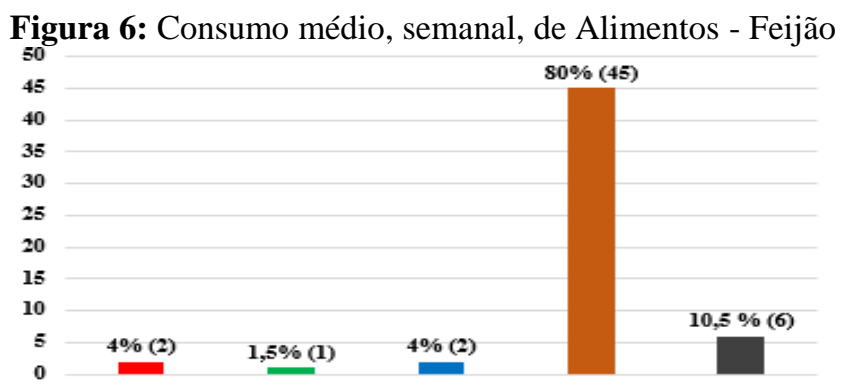

Feminino / 2-3x por semana

Feminino / 4-6x por semana

Masculino - 4-6x por semana

Feminino - Todos os dias

Masculino - Todos os dias

Fonte: os próprios autores.

De acordo com o Ministério da Saúde, é recomendado a ingestão de feijão, na dieta alimentar, pelo menos uma vez por dia, quatro vezes por semana, no mínimo. 12

Trabalho realizado no Rio Grande do Sul, $71 \%$ dos entrevistados relataram seguir as recomendações expressas pelo Ministério da Saúde, porém a maioria da população brasileira não possui este hábito. ${ }^{13}$

A figura 7 analisa o consumo médio, semanal, de alimentos gordurosos, embutidos e frituras: carne com gordura aparente, salsicha, frituras, salgadinhos e embutidos. As respostas obtidas mostram que a maioria entrevistados 59 $\%$ faz uso desses alimentos duas a três vezes por semana do publica feminino; $10,5 \%$ faz uso desses alimentos duas a três vezes por semana do publica masculino; $10,5 \%$ quatro a seis vezes por semana do público feminino; $4 \%$ quatro a seis vezes por semana do público masculino; $16 \%$ menos de uma vez por semana.

Figura 7: Consumo médio, semanal, de Alimentos gordurosos, embutidos e frituras

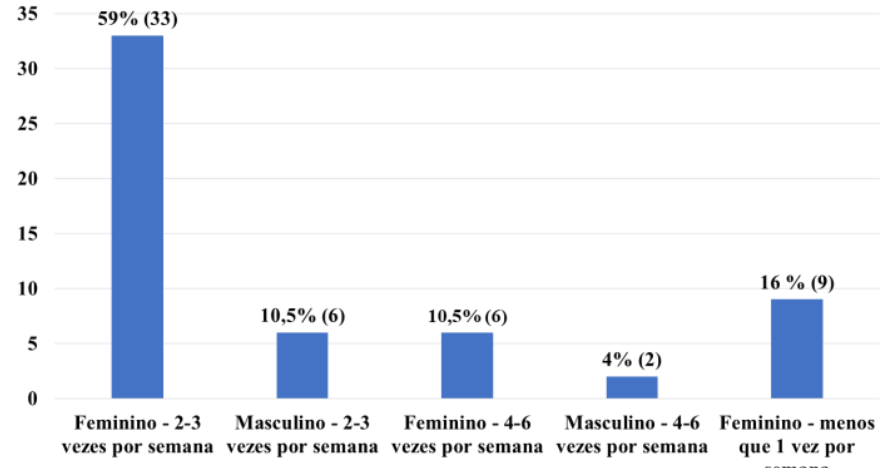

Fonte: os próprios autores.
Quanto ao hábito alimentar, referente ao consumo de embutidos e alimentos gordurosos, o Ministério da Saúde recomenda o uso de, no máximo, uma vez por semana. ${ }^{16}$ Os dados apresentados está de acordo com a prevalência de excesso de peso na população idosa, principalmente do sexo feminino. ${ }^{15}$

A figura 8 analisa o consumo médio, semanal, de bebidas- refrigerante usando as opções de $300 \mathrm{ml}$ pelo menos de uma vez por semana; duas a três vezes por semanas por semana; quatro a cinco vezes por semanas e todos os dias. Os resultados obtidos mostram que a maioria do público idoso, em especial o público feminino faz uso de duas a três vezes por semana e valor superior a $300 \mathrm{ml}$ na semana. Seguido por $37,5 \%$ duas a três vezes na semana do público feminino; $10,5 \%$ duas a três vezes na semana do público masculino; $2 \%$ quatro a seis vezes na semana do público feminino; 35,5\% menos que uma vez por semana do público feminino; $4 \%$ menos que uma vez por semana do público masculino e $10,5 \%$ não faz uso todos os dias o público.

Figura 8: Consumo médio, semanal, de Bebidas - Refrigerantes $300 \mathrm{ml}$ 25

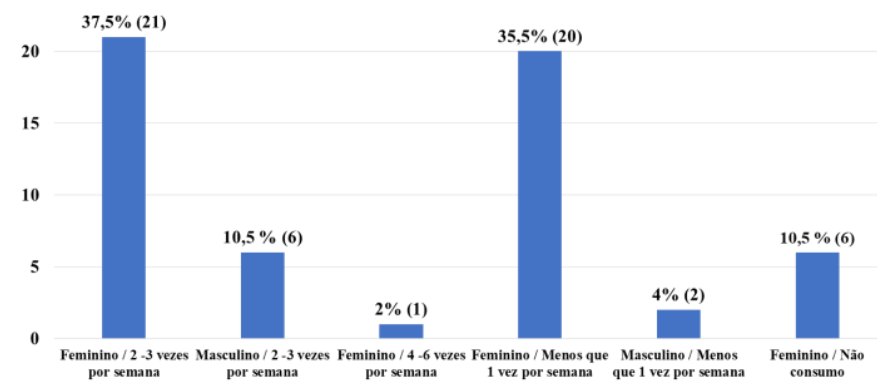

Fonte: os próprios autores.

O uso continuo de refrigerantes, não é recomendável nos alimentos e bebidas saudáveis descritas pelo Ministério da Saúde. Trabalho realizado por Vinholes, Assunção e Neutzling, 20\% dos entrevistados consomem, diariamente, refrigerantes. Os dados apresentados acima, é nítido que o sexo feminino possui o hábito de não ingerir refrigerante com alta frequência. ${ }^{15,16}$

Em relação ao hábito de adicionar sódio nos alimentos previamente temperados, $55 \%$ do público feminino não fazem adição, já os idosos do sexo masculino apenas 7,5\% não fazem adição de sódio (Figura 9). 
Figura 9: Adição de sódio nos alimentos previamente temperados

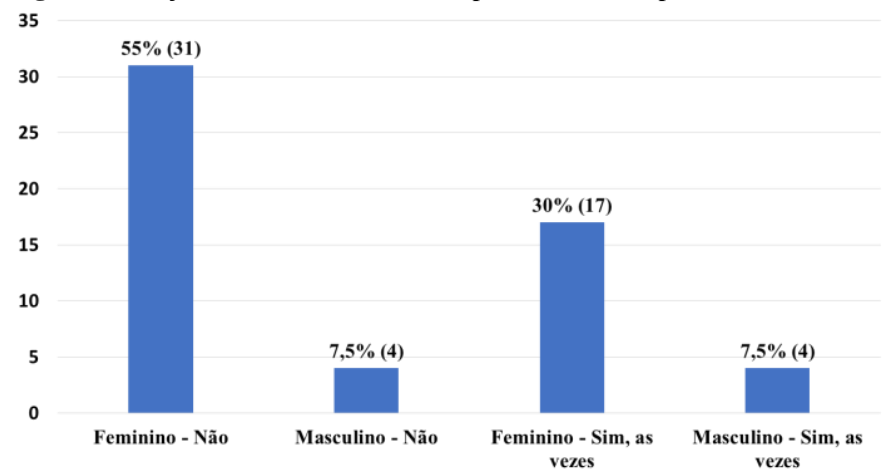

Fonte: os próprios autores.

A redução do consumo de sal é considerada um dos passos iniciais e fundamental medida não farmacológica do manejo terapêutico do público sujeito a patologia de hipertensão, sendo recomendado para esses indivíduos o consumo de, no máximo, 4g/sal/dia ou 1600mg/sódio/dia (5). Contudo, estudos recentes evidenciam consumo de sal em torno de 12,5 a 13g/dia, entre hipertensos, dado majoritariamente pela adição de sal no preparo dos alimentos (6-7). ${ }^{17}$

Quanto a análise do consumo de alimentos ricos em carboidratos, de duas a três vezes por semana o público feminino respondeu por $27 \%$, de duas a três vezes por semana o público masculino respondeu por $9 \%$, de quatro a seis vezes por semana o público feminino respondeu por $16 \%$, de quatro a seis vezes por semana o público masculino respondeu por $2 \%$, menos que uma vez por semana o público feminino respondeu por $28,5 \%$, todos os dias por semana o público feminino respondeu por $14 \%$ e todos os dias por semana o público masculino respondeu por $3,5 \%$.

Figura 10: Consumo de alimentos ricos em carboidratos (massas, doces, biscoitos, etc.)

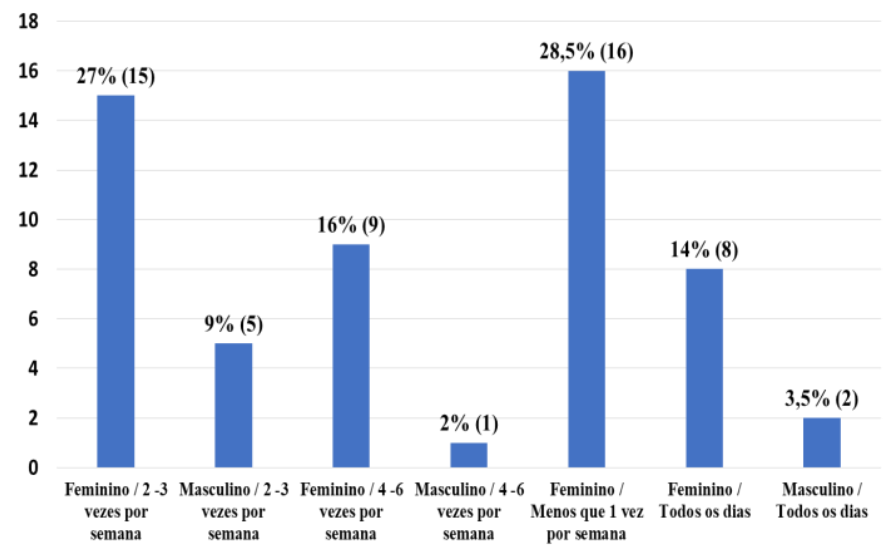

Fonte: os próprios autores.
O conhecimento do metabolismo dos carboidratos e sua relação com a elevação glicêmica, em seus aspectos qualitativos e quantitativos é enfatizada por possibilitar um bom controle, principalmente no período pós-prandial. É comentada também a correta utilização de proteínas para prevenir ou tratar nefropatia e gorduras para evitar a dislipidemia, obesidade e doença cardiovascular. Sacarose e edulcorantes artificiais devem ser utilizados com critérios.

As características da dieta nas diferentes fases da evolução do diabetes mellitus tipo $1 .{ }^{18}$

A figura 11 analisa a quantidade diária de refeições que são feitas por dia pelos idosos usando de três até quatro refeições. A maioria dos entrevistados faz quatro refeições por dia $55 \%$, seguido de três vezes por dia do público feminino $41 \%$, três vezes por dia do público masculino $4 \%$ três vezes por dia.

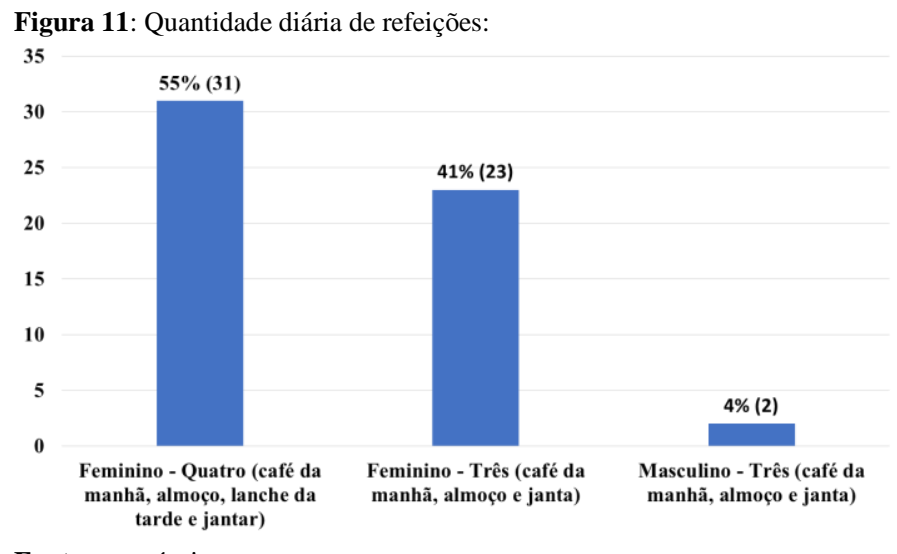

Fonte: os próprios autores.

É recomendado que toda pessoa faça, pelo menos, quatro refeições diariamente, considerando três refeições principais e um lanche por dia. Conforme os dados acima, todos os idosos entrevistados possuem hábito alimentar de três refeições diárias, pelo menos. ${ }^{15}$

Na questão do tabagismo o número de idosos que não costuma fazer uso de tabaco foi soberano. Dos 56 entrevistados, $71 \%$ não faz uso de cigarro do público feminino, $6 \%$ não faz uso de cigarro do público masculino, $14 \%$ não usa cigarro, mas já fumou relacionado ao público feminino e $9 \%$ do público masculino não fuma, mas já fumaram (Figura 12). 
Figura 12: Foi ou atualmente é fumante?

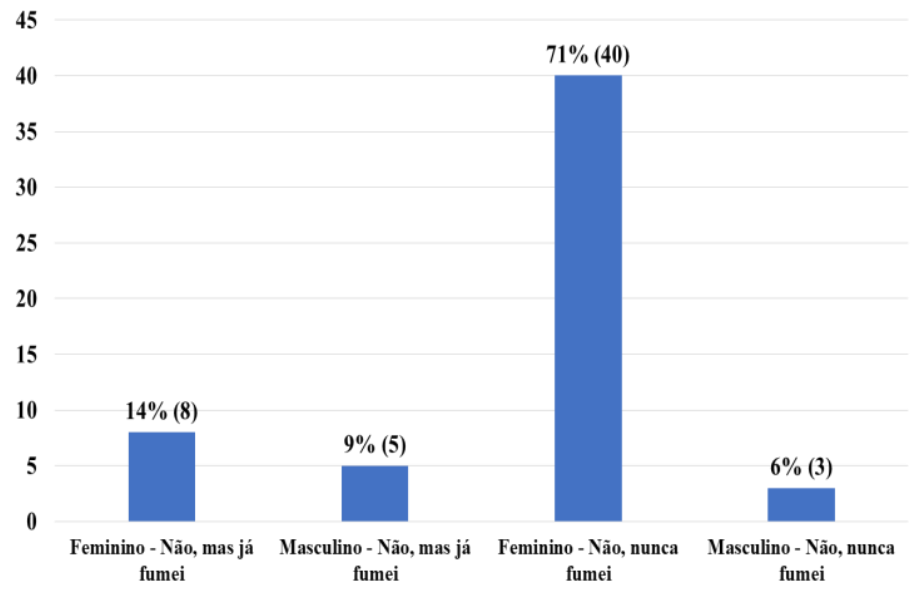

Fonte: os próprios autores.

Todos os idosos, atualmente, não são fumantes. Já trabalho publicado em 2012, apenas 35\% dos idosos entrevistados não são fumantes. ${ }^{19}$ Porém, Zaitune e colaboradores, o número de idosos brasileiros fumantes está reduzindo, principalmente pelas orientações recomendadas pelos profissionais da saúde que orientam sobre diabetes e hipertensão. ${ }^{20}$

A figura 13, demonstra o baixo consumo de álcool foi visível na entrevista realizada no público idoso. 3,5\% do público feminino usam de duas a três vezes por semana, $7 \%$ do público masculino usam de duas a três vezes por semana, $23 \%$ do público feminino usam uma vez por semana, $5 \%$ do público feminino usam uma vez por semana, $60 \%$ do público feminino não usam e 1,5\% do público masculino não usam.

Figura 13: Consumo médio (600mL), semanal, de Bebidas - Alcoólicas

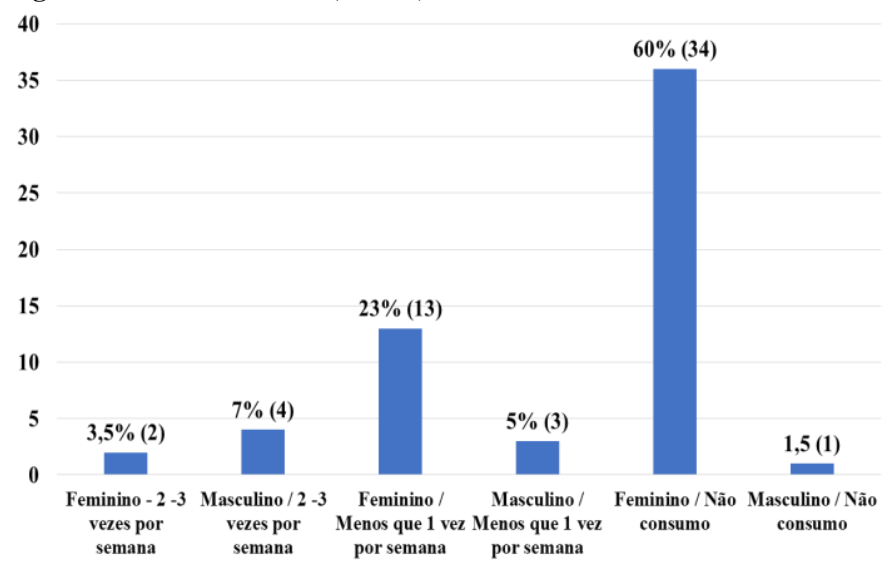

Fonte: os próprios autores.

Ferraz, Reis e Lima relataram que $88 \%$ dos idosos entrevistados não consomem bebida alcoólica. Estes dados corroboram com os resultados, representados na figura 14 , onde a maioria dos idosos não possuem o hábito de ingerir bebida alcoólica constantemente. ${ }^{21}$
$\mathrm{Na}$ figura 14 teve como discussão o Índice de massa corporal-IMC dos entrevistados, para isso fizemos o uso de dados, como peso e altura para aplicar na formula adequada. Assim, foi observado que $62 \%$ dos entrevistados, do público feminino, estão com IMC acima do peso, $10 \%$ do público masculino estão acima do peso, 3,5\% do público masculino estão com peso normal e $23,3 \%$ do público feminino estão om o peso normal.

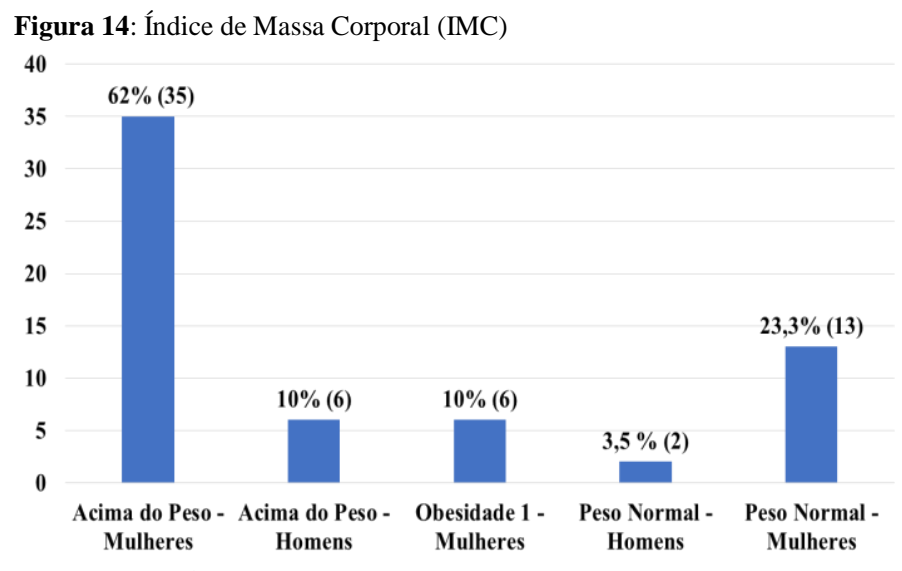

Fonte: os próprios autores.

Figura 15: Cardeneta de Saúde da Pessoa Idosa (IMC), Brasil 2014.

\begin{tabular}{c|l|l}
\hline Índice antropométrico & \multicolumn{1}{|c|}{ Pontos de corte } & \multicolumn{1}{c}{$\begin{array}{c}\text { Classificação do estado } \\
\text { nutricional }\end{array}$} \\
\hline \multirow{2}{*}{ IMC } & $<22 \mathrm{~kg} / \mathrm{m}^{2}$ & Baixo peso \\
\cline { 2 - 3 }$\frac{\text { Peso }}{(\text { Altura })^{2}}$ & $\geq 22 \mathrm{e} \leq 27 \mathrm{~kg} / \mathrm{m}^{2}$ & Peso adequado \\
\cline { 2 - 3 } & $>27 \mathrm{~kg} / \mathrm{m}^{2}$ & Sobrepeso \\
\hline
\end{tabular}

O Índice de Massa Corporal (IMC) está diretamente relacionado ao hábito de atividades físicas e o sedentarismo. O IMC dos entrevistados, no trabalho realizado em 2009, $46 \%$ estão com o índice adequado pelo tamanho e altura dos indivíduos. ${ }^{13}$

$\mathrm{Na}$ figura 16, os antecedentes familiares para HAS. Dos 56 entrevistados, $42,5 \%$ do público feminino responderam que não há alguns parentes de primeiro grau com HAS, 5\% do público masculino responderam que não há alguns parentes de primeiro grau com HAS, 42,5\% do público feminino responderam que há alguns parentes de primeiro grau com HAS e $10 \%$ do público masculino responderam que há alguns parentes de primeiro grau com HAS. 
Figura 16: Parentesco de primeiro grau diagnosticado com Hipertensão

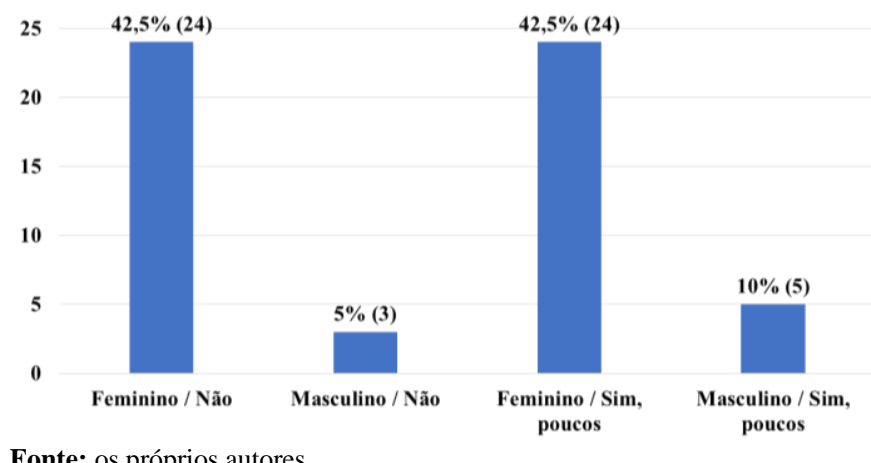

Fonte: os próprios autores.

De acordo com a figura 17, os antecedentes familiares para Diabetes Mellitus. Dos 56 entrevistados, 29\% do público feminino responderam que não há alguns parente de primeiro grau com Diabetes Mellitus, 1,5\% do público masculino responderam que não há alguns parente de primeiro grau com Diabetes Mellitus, 7\% do público feminino responderam que há sim, a maioria dos parentes de primeiro grau com Diabetes Mellitus e 53,5\% do público feminino responderam que há sim, a poucos parentes de primeiro grau com Diabetes Mellitus e 9\% do público masculino responderam que há sim, a poucos parentes de primeiro grau com Diabetes Mellitus.

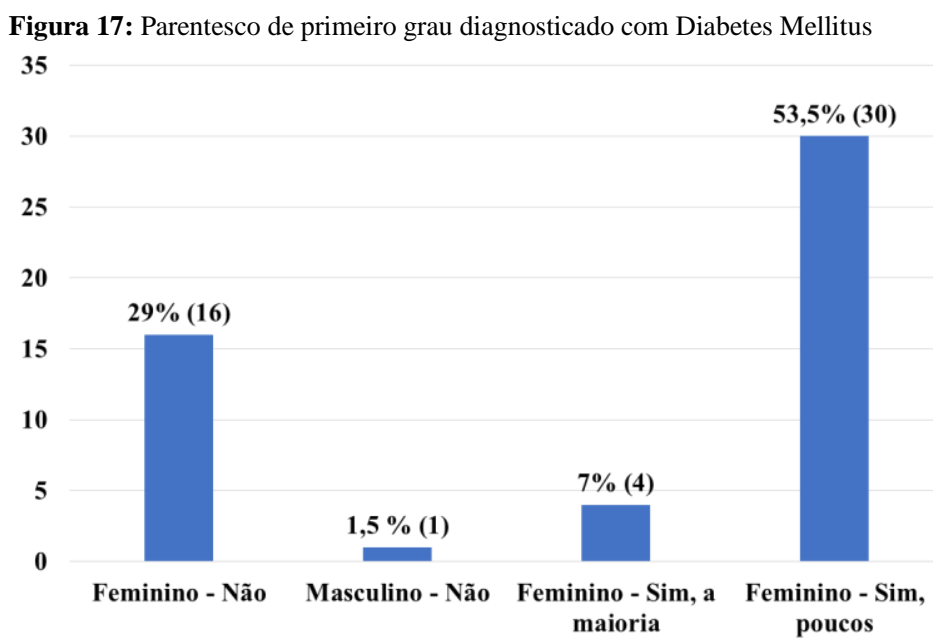

Fonte: os próprios autores.

O maior número de hipertensos, na população brasileira, são os idosos. ${ }^{22}$ Ferraz, Ferreira e Lima relataram que $28,3 \%$ dos idosos entrevistados relataram ser portados de Diabetes e Hipertensão. Porém, diversos trabalhos relaram que idosos portadores de DM buscam a melhoria da qualidade de vida, prática de exercícios físicos, além da alimentação regulada e restrita. ${ }^{23,25,25}$

\section{CONCLUSÃO}

Com os resultados deste trabalho, é possível concluir que os idosos participantes da pesquisa possuem hábitos alimentares regularmente saudáveis que auxiliam contra doenças crônicas como Diabetes Mellitus e HAS. Porém, o fator de risco genético mostrou-se relevante e o Índice de Massa Corporal da maioria está acima do peso ou em obesidade I.

É válido ressaltar, que a prevalência de diabetes mellitus, mesmo sendo menor em homens, não é condizente com o curso clínico desta, pois são neles a prevalência dos casos mais graves e com pior prognóstico da doença, visto que não possuem hábitos para o controle e manejo desta morbidade clínica.

\section{REFERÊNCIAS BIBLIOGRÁFICAS}

${ }^{1}$ SKYLER, J. S. Diabetes mellitus: Pathogenesis and treatment strategies. Journal of Medicinal Chemistry 2004, 47, 4113.

${ }^{2}$ FERREIRA, V. A.; CAMPOS, S. M. B. Avanços farmacológicos no tratamento do diabetes tipo 2. Brazilian Journal of Surgery and Clinical Research 2014, 8, 72.

${ }^{3}$ WILDING, J. P. H. The role of the kidneys in glucose homeostasis in type 2 diabetes: Clinical implications and therapeutic significance through sodium glucose cotransporter 2 inhibitors. Metabolism 2014, 63, 1228.

${ }^{4}$ MORAES SA, FREITAS ICM, GIMENO SGA, MONDINI L. Diabetes mellitus prevalence and associated factors in adults in Ribeirão Preto, São Paulo, Brazil, 2006: OBEDIARP Project. Cad Saúde Pública 2010; 26(5): 929-41.

${ }^{5}$ SOUZA LJ, CHALITA FEB, REIS AFF, TEIXEIRA CL, GICOVATE NETO C, BASTOS DA, et al. Prevalence of diabetes mellitus and risk factors in Campos dos Goytacazes, RJ. Arq Bras Endocrinol Metab 2003; 47(1): 69 74.

${ }^{6}$ LYRA R, SILVA RS, MONTENEGRO JR RM, MATOS MVC, CÉZAR NJB, MAURÍCIO-DA-SILVA L. Prevalence of diabetes and associated factors in an urban adult population of low educational level and income from the Brazilian Northeast wilderness. Arq Bras Endocrinol Metab 2010; 54(6): 560-6.

${ }^{7}$ SECRETARIA DE SAÚDE DO ESTADO DE GOIÁS. Conscientização e prevenção são armas contra diabetes. Jun/2019. Disponível em <http://www.saude.go.gov.br/conscientizacao-e-prevencao-sao-armas-contradiabetes/> acessado em 04/11/2019.

${ }^{8}$ BRASIL. Ministério da Saúde, 2001. Cadernos de Atenção Básica - caderno 7, Hipertensão Arterial Sistêmica (HAS) e Diabetes mellitus (DM). Brasília 2001

${ }^{9}$ FARIA CC e CARLOS BR. Atividade física para prevenção e tratamento das doenças crônicas não transmissíveis e da incapacidade funcional. Rev. Nutr. 2009

${ }^{10}$ SAPERSTEIN A \& PENNER AM. (2012). Racial Fluidity and Inequality in the United States. American Journal of Sociology, 118(3), 676-727.

${ }^{11}$ SANTOS RD, GAGLIARDI ACM, Xavier HT, MAGNONI CD, CASSANI R, LOTTENBERG AMP, et al. I Diretriz sobre o consumo de gorduras e saúde cardiovascular. Arquivos Brasileiros de Cardiologia. vol. 100, nº1, Supl. 2, Jan 2013 . 
12 BRASIL, Ministério da Saúde. Secretaria de Atenção à Saúde. Departamento de Atenção Básica. Diabetes Mellitus / Ministério da Saúde, Secretaria de Atenção à Saúde, Departamento de Atenção Básica. - Brasília: 2006. 64 p. il. Cadernos de Atenção Básica, n. 16.

${ }^{13}$ BOTELHO VD, FORMOSO MC, BORGES NM. Frequência de hábitos saudáveis de alimentação medidos a partir dos 10 Passos da Alimentação Saudável do Ministério da Saúde: Pelotas, Rio Grande do Sul, Brasil. Cad. Saúde Pública. 2009 Apr

${ }^{14}$ LEVY-COSTA RB, SICHIERI R, PONTES NS, MONTEIRO CA.

Household food availability in Brazil: distribution and trends (1974-2003). Rev Saúde Pública 2005; 39:530-40.

${ }^{15}$ Instituto Brasileiro de Geografia e Estatística. Pesquisa de Orçamentos Familiares 2002-2003: análise da disponibilidade domiciliar de alimentos e do estado nutricional no Brasil. Rio de Janeiro: Instituto Brasileiro de Geografia e Estatística; 2004.2008; 17:310-4.

${ }^{16}$ BRASIL. Ministério da Saúde. Secretaria de Atenção à Saúde. Departamento de Atenção Básica. Guia alimentar para a população brasileira. - 2. ed., 1. - 2014. 156 p.

${ }^{17}$ AGONDI RF, GALLANI MCBJ, CORNÉLIO ME, RODRIGUES RCM. Análise dos planos de ação e planos de enfrentamento de obstáculos para a redução do consumo de sal entre mulheres com hipertensão arterial sistêmica. Rev. Latino-Am. Enfermagem. 2012

${ }^{18}$ LOTTENBERG AMP. Características da dieta nas diferentes fases da evolução do diabetes mellitus tipo 1. Arq Bras Endocrinol Metab. 2008

${ }^{19}$ ZAITUNE MPA, BARROS MBA, LIMA MG, CÉSAR CLG, CARANDINA L, GOLDBAUM $M$ et al . Fatores associados ao tabagismo em idosos: Inquérito de Saúde no Estado de São Paulo (ISA-SP). Cad. Saúde Pública. 2012

${ }^{20}$ LUCOVEIS MLS, Gamba MA, Paula MAB, Morita ABPS. Degree of risk for foot ulcer due to diabetes: nursing assessment. Rev Bras Enferm [Internet]. 2018;71(6):3041-7.

${ }^{21}$ FERRAZ, M., DE, O., S; REIS, L., A., DOS; LIMA, P., V. Condições de saúde de idosos portadores de Diabetes mellitus e hipertensão arterial sistêmica. Rev. Psic. v.10, n. 33, 2017.

${ }^{22}$ GRAVINA CF, GRESPAN SM, BORGES JL. Tratamento nãomedicamentoso da hipertensão no idoso. Rev Bras Hipertensão 2007;14(1):33-6.

${ }^{23}$ GARCIA LPRR e TAVARES SAO. Mortalidade por Diabetes Mellitus em Goiás no período de 2008 a 2015. Boletim Epidemiológico. Goiás. Vol 18 No5. Disponível em: http://www.sgc.goias.gov.br/upload/arquivos/2018 07/boletim-epidemiologico---diabetes.pdf. Acesso em: 26 de Fevereiro de 2019

${ }^{24}$ DIRETRIZES DA SOCIEDADE BRASILEIRA DE DIABETES 2017-2018. Disponível em:

https://www.diabetes.org.br/profissionais/images/2017/diretrizes/diretrizessbd-2017-2018.pdf. Acesso em: 26 de Fevereiro de 2019.

${ }^{25}$ ZANCHIM MC.; KIRSTEN VR., MARCHI ACB. Marcadores do consumo alimentar de pacientes diabéticos avaliados por meio de um aplicativo móvel. Ciênc. saúde coletiva, Rio de Janeiro, v.23, n.12, p. 4199-4208, Dec. 2018. 